\title{
The Impact of Perception Difference on Channel Conflict: A Customer Relationship Life Cycle View
}

\author{
Lan Wang \\ School of Business, Beijing Language and Culture University, Beijing, China \\ Email: im2003@163.com
}

Received 8 July 2015; accepted 14 September 2015; published 17 September 2015

Copyright (c) 2015 by author and Scientific Research Publishing Inc. This work is licensed under the Creative Commons Attribution International License (CC BY). http://creativecommons.org/licenses/by/4.0/ c) (i) Open Access

\begin{abstract}
In different phases of the life cycle in client relations, the importance level of influential factors for channel conflicts varies because of the differences in communication methods and trust levels between the two ends. This thesis has clearly defined the research scenario-at the mature phase of client relations, to study how perception difference on channel conflict. We argue that the higher level of the perception difference is, the higher level of channel conflict is. At the period of mature client relations, both sides should lessen the perception difference, communicate timely, improve the mutual understanding of fairness in relations, and build complete clients' satisfaction and trust.
\end{abstract}

Keywords

Perception Difference, Channel Conflict, Maturity Period

\section{Introduction}

There are many discussions about channel conflicts in recent years. Professional magazines have published a lot of articles concerning about channel conflicts, and most of these articles analyzed specific problems about channel conflicts in the view of channel operation and management. Although the suggestions from those articles are useful for channel conflicts management in some traditional industries, they are not theoretically sufficient to analyze systematically about the appearing process and management of channel conflicts.

In order to create a rather high standard relation value, it is needed to construct efficient marketing channels that operate harmoniously to manage conflicts effectively. Based on the background from above, and researches of related literatures, this thesis attributes to discuss the influence of perception difference between manufactur- 
ers and retailers to channel conflicts under the particular market environment and social background in China at the phase of mature client relations.

In different phases of the life cycle in client relations, the importance level of influential factors for channel conflicts varies because of the differences in communication methods and trust levels between the two ends. This thesis has clearly defined the research scenario-at the mature phase of client relations, to research the vertical channel conflicts between manufacturers and retailers from retailers' point of view-to make the results more targeted and precise. Moreover, this is an attempt to overcome the defect that current researches cannot reveal the dynamics of the factors in channel conflicts.

According to client relation theory, the optimum client relation mode under the principle of profit maximization is to shorten the periods of observation and formation, and to extend the period of maturity as long as possible [1]. It can be seen that the most important and valuable phase of client relations is the maturity period, but it is also the most dangerous phase. Channel members must adopt continuous measures to strengthen channel relations; otherwise the relations will enter recessions very easily [2].

Knowing how to reduce and solve conflicts is very important for channel members to enhance cooperation and build mutual trust, and it is the reason why this thesis focuses on the maturity period. A thorough analysis of the causes of conflicts can be taken as an early warning mechanism for conflicts. It is of great significance to take preventive measures before conflicts appear, and solve the existing conflicts before they become damaging.

\section{Literature Review}

Life cycle is a very useful tool in researching dynamic features in client relations. The maintenance of relations has a dynamic nature, and conflict is one type of client relations, therefore the researches of channel conflicts should embody dynamic features. Truly, conflicts can be triggered by many factors, while some of them are dominating and the others are minor. It is only by clearly understanding the relation between conflicts and the levels of channel conflicts can people see the essence through facts, and therefore encourage the establishment of an effective mechanism for conflicts management.

Rosenberg and Stem (1970) developed the channel conflicts model according to Pondy's organizational conflicts model—conflict reasons - conflict behaviors-conflict results (shown as the performances of channel members). According to the primary model by Pondy, Friat defined conflicts as a process from cognitive conflicts to the manifest of opposite behaviors. At the former phase of conflicts, channel members demonstrate emotions of frustration and hostility [3]. However, at the last phase of conflicts, channel members are not satisfied by just showing negative emotions but adopting revenging behaviors and using legal arbitration. On some extreme circumstances, channel members might leave the channels and persuade other members to leave, which would hugely affect channel cooperation, trust and efficiency. It is rather difficult to distinguish the different states within a process (Schmidt and Kochan, 1972). In fact, most of the researches about channel apply the principles of dichotomy - dividing the conflicts into potential conflicts and emerging conflicts according to the emergence degree of conflicts. Stem and El-Ansary (2001) claimed that a channel conflict is a disharmonious and hostile mode when one side of the conflict considers that the other side is hindering it from achieving goals [4].

Most of the scholars hold relatively consistent opinions about the reasons of channel conflicts. They believe that conflicts are inherent phenomena in channels because channel members are functionally dependent (Assael 1968, Gorman 1969, Cadotte and Stem 1979, Lusch 1976, Pondy 1967, Reve and Stem 1979, Stem and ELAnsary 1977). Channel conflicts might originate from more specific functional relations. Etgar (1979) stated that, in principle, some structural factors such as inconsistent targets, need of independence and control lead to dominate conflicts. Stem concerned that differences of objects, opinions about facts, and fields are the main reasons for channel conflicts. Field difference includes four elements: people as the serving objects, the covered area, tasks needed to be finished, related technical problems in marketing [5]. Gattorna (1978), Rosenberg and Stem (1970) have proved that the bigger the target divergence, the smaller the difference of region, the bigger the misunderstanding of the fact lead to more channel conflicts [6].

Michman and Sibley (1980) have announced five factors that are possible to affect channel conflicts from the integrated models of channel conflicts: environment, channel structural nature, channel mode, the positioning of channel members, and the industry standard. These five factors contain micro and macro factors. Macro factors such as environment factor cannot be controlled by channel members. Some of the factors will enhance the co- 
hesion of channels, while some factors will cause tension and conflicts among channel members. Schul and Babakus used the channel structure as an intermediate variable to build up the relation model between power and conflicts. They have also conducted researches in chartered real estate agents, and the results showed that the effect on conflicts from remuneration right and enforcement power depends on channel members' perceptions of the channel decision-making structures. It is proved that remuneration right and enforcement power have no direct connections, but they function through the intermediate variable-the decision-making structure, which is different from former results [7]. Etgar (1979) divided conflicts into potential and emerging phases to research, and categorized the factors of conflicts into structural and subjective groups. After researches of six different industries, it is concluded that both potential conflicts and emerging conflicts are influenced by subjective factors (perception difference, expectation difference, role obscurity, spreading obstacles, etc.).

Western scholars started the researches about channel conflicts rather early, and the recent researches are mainly about channel benefits, channel organizational systems and mode, channel behaviors, the new relations of channels, the internet and channel innovations. The theory and practice of marketing channels are becoming to be people's focus. As for channel conflicts, Zhuang Guijun and other scholars studied about the relation among channel rights, conflicts and cooperation, and have made conclusions that are rather consistent with western researches [6]. They also applied dyadic approach to analyze the influences on conflicts caused by the perception difference of dyadic channel members. It is shown that there is a big difference of the mutual dependence perception of channel relations from the both sides of channel members, and this difference will cause the difference on behaviors.

According to the literature review above, it can be concluded that, firstly, the current researches about the factors of channel conflicts are done by western scholars based on western marketing practices. Therefore, the results may not be suitable for the marketing channels in Chinese market. Secondly, the nature of conflicts is a relation, and the researches of relations are dynamic. Current researches are relatively vague, so it is needed to divide different relation phases, in order to deepen and systematize the researches. There are many factors that influence the channel conflicts, while some of the factors raised by different scholars are overlapping. Hence, the factors can be categorized into macro and micro factors. Rosenberg (2002) summarized the reasons of conflicts in channels according to researches of the literatures. He stated that the reasons of conflicts can be generalized into one or more than one of the following basic aspects: opposition of roles, scarcity of resources, perception difference, expectation difference, divergence in decision-making area, inconsistent targets, and obstacles in communication [8]. This thesis discusses specifically about the influences caused by perception difference on channel conflicts at the mature phase of client relations.

\section{The Influences of Perception Difference on Channel Conflicts}

\subsection{Research Scenario}

In order to make researches deeper and more detailed, a clear research scenario should be set about the relation. Channel conflict itself is a type of marketing channel relation, so the study of conflicts must have a clear research scenario. In many of the marketing channel definitions, Bucklin's (1966) and Stem's (1969) definitions reflect the features of marketing channels more thoroughly and systematically. Bucklin (1969) defined the concept in the view of components in marketing channels. He believed that marketing channels are consisted by two main subsystems (or components). These two subsystems are business subsystem and product user subsystem. The business subsystem is comprised by a series of vertical mutual related marketing organizations or institutes, including manufacturers, wholesalers and retailers. On the other hand, product user subsystem contains families, industries or institutes, and they build up a task environment for the marketing channel.

It can be seen that the business subsystem, which is a vertical chain combined by manufacturers, wholesalers, and retailers is the main body of the marketing channel. The components of the business subsystem provide available services for the product user subsystem (a task environment combined by families, industries or institutes) as a whole. Therefore, it is easy to understand why most of the marketing channels only define their business subsystems. Among them, Stem's (1969) definition is more representative-the marketing channel is a group of interdependent organizations that promote the usage and consumption of a product or service by exchanges of productions.

Therefore, it is possible to define the structure of the marketing channel: the structure of marketing channel is the way that the main bodies of the marketing channel (manufacturers, wholesalers and retailers, etc.) are orga- 
nized and interconnected. Most of the studies of marketing channel relations focused on the formation of channel trade organizations, and it is also called as the dyadic relationship structure management of channels. There are many formations exist in between of manufacturers and wholesalers, manufacturers and retailers, wholesalers and retailers in relations studies, including department stores, shopping malls, supermarkets, exclusive agencies, convenience stores, and franchised stores, etc. Along with the growth of the amount of retailers, more and more retailers trade directly with manufacturers because the channel power moves downward. For the studies of conflicts between manufacturers and retailers, there are three angles: the perception of conflicts from manufacturers' view, the perception of conflicts from retailers' view, and collect matching statistics to research the perception difference towards conflicts. This thesis will research the conflicts between manufacturers and retailers from retailers' view.

\subsection{Perception Difference and the Level of Channel Conflicts}

Perception difference: Perception difference is the process of choices and explanation of people toward external stimulations. For the same object, different people will acquire different phenomenal experience, which leads to different perceptions [9]. Generally, the ways to perceive stimulations are very different from facts. A perceived object is a phenomenal experience in one's mind, although it reflects the object itself on some degree, it could be "right" (when it reflects the object correctly) or "wrong" (when it reflects the object not correctly enough). In marketing channels, different members might perceive the same stimulus but have very different explanations. These perceptions include the perception of dependence, fairness, industry environment, the degree of market competition. Researchers of channel theories are interested in the differences of perceptions and their influences [10]. From existing researches, perception difference is an important factor that causes potential conflicts. Domestic scholars studied six department stores in Xi'an. They researched the relation between dependent perception difference and the level of channel conflicts by using dyadic approach recommended by Gaski. The results proved the existence of perception difference of dependence among channel members. The dependent relation between channel members is an objective reality, but it is also a person's subjective awareness or perception status.

The perception difference of dependence: The perception difference of dependence can cause conflicts or arguments among channel members in the following way. When A thinks that the dependence level of itself towards B is low, but B believes that the dependence level from A is high. Therefore, A does not expect B to influence its decision-making and behaviors under enforcement power, whereas $B$ expects a low resistance when it uses its power to influence A's decision-making and behaviors. Because of the perception difference, B might utilize powerful behaviors that are unacceptable for A to influence A, and A might response B with a power that B cannot accept. When one side of the channel uses a power that excesses the other side's acceptance to influence the other side's decision-making or behaviors, and the other side responses with a power that is unacceptable, conflicts and arguments exist. The higher the perception difference of dependence is, the higher the channel members' perception level of conflicts [11].

The perception of fairness is channel members' understanding of fairness about the vertical and horizontal relations. Dealers themselves distinguish whether manufacturers are fair to the same-level dealers, including the price of products, promotion supports, punctuality of deliveries, etc. If dealers think that the value (revenue/cost) they get from the relation is the same or higher comparing to the value the suppliers get from the relation, the clients would agree that the relation is fair. Fairness theory and social trade theory studied and believed that the output of fair (or unfair) relations will influence the behaviors of evaluators [12]. Fair outputs of relations make the dealers to believe that manufacturers care for the benefits of dealers, so the level of relation opportunism declines. On the contrary, if dealers think that they are involved in an unfair relation, they would consider themselves being bullied by manufacturers, which would lead to anger and potential conflicts. The negative emotions toward manufacturers will cause disbeliefs and doubts [13]. Hence, the higher level of the perception difference is, the higher level of channel conflict is, and vise versa.

\subsection{Case Analysis: The Effective Communication Mechanism of Company a Reducing Perception Difference}

Company A provides supplier chain integration services for high-tech industries. Its business spreads to more than 20 countries and regions around the world. The products of company A include information, communica- 
tion, consuming electronics and element components, offering clients a variety of brands and products and onestop shopping experience. As a member of the brand channel, company A integrates selling, channel and post-service.

One of the strategic principles company A adopts is to maintain an impartial role in the channel, focusing on premium channel services, as well as creating values and reducing costs for manufacturers and dealers, avoiding competitive relations with the served companies. In order to improve the quality of services, company A centralizes the needs of clients during the entire process, offering comprehensive and individualized services from price enquiries to the final post-service. A mature process of supplier chain management and the informatization of the process are important factors for company A to offer integrated supplier chain services. This mature process could guarantee the communications and the integration of resources across job responsibilities and companies. By specialized and differentiated logistical services, company A overviews the entire supply chain and concentrates on the needs of the clients, to form closer relations with the upstream suppliers and the downstream clients. Company A develops long-term oriented relations with clients, builds up effective communication mechanism, and develops win-win cooperation by reducing perception difference.

From the view of corporative collaboration and development process, due to the companies' mission and the requirements of work division, the primary interaction between companies is because of the requests about job responsibilities and the needs of work, i.e. the working relations people are familiar with. Meanwhile, it is significantly important for both sides (supplier and purchaser) to establish communication channel that can express respective expectation and share valuable information/knowledge when operating business and collaborations to reduce perception difference.

In this formal communication mechanism, the format and contents of the interactions between company A and suppliers include cross-functional groups, formal communications, and regular structural meetings. The cross-functional groups across companies and the regular meetings with suppliers can provide effective institutional guarantee for the improvements of the quality of interactions within and between organizations, and the performance of the supply chain. In addition, the purpose of establishing cross-functional groups is not only for transmitting valuable knowledge and information in time, but also for uniting the values of both sides (supplier and purchaser) and the cultural system in enterprises. Except for the mandatory requirements such as agreed prices and related regulations in contracts and agreements, flexible constrains such as supplementary information must be implemented by informal networks. Generally, when senior employees of both enterprises, or the vice presidents/managers of both the purchasing department in the purchasing company and the the selling department in the supplier company come to an agreement or a basic framework of an idea, practical operations are done by general employees. These interactions are usually about crafts, skills or knacks. The operators from both sides can reduce the obstacles of business-related communications and the perception differences by daily informal interactions, such as after-work meetings and entertainments among friends. Also, the interaction history of both companies can help them to decode the special language of the opposite company and understand the tacit knowledge, to improve the quality of formal interactions.

It is worth saying that, the supplier and purchaser are connected because of the division of work in the industrial chain. The resources processed respectively by both sides determine that the two subjects need to interact in a certain way, creating values to meet the needs of clients and gain their satisfaction. In the process of collaboration, both of the supplier and the purchaser are motivated to maximize their profits. Therefore, they cannot guarantee the best results of the entire process, but rather intensify the contradictions and increase the obstacles of collaboration, betraying the primary goal of a close cooperation. It is to say that, the formal and informal interaction mechanisms between suppliers and purchasers are only the foundation of collaboration, but it cannot ensure the predictive outcomes. In order to guarantee the maximization of overall benefits, the respective behaviors should be coordinated and regulated, to get rid of selfish opportunistic behaviors.

\section{Result Analysis}

At the mature period of client relations, both sides must keep the innovative ability of continuous increase and offer personalized additional services to establish the sustainable client loyalty. Because the perceptions of dependence and fairness of the both sides directly influence the degree of client's satisfaction, but the accumulation of dissatisfaction lead to tensions to relations, causing conflicts. At the period of mature client relations, both sides should lessen the perception difference, communicate timely, improve the mutual understanding of 
fairness in relations, and build complete clients' satisfaction and trust. At the period of mature client relations, as the understanding of alternative manufacturers increased and the self-value evaluation level upgraded, clients' horizons have been broadened, and the value evaluation started to shift from internal comparisons to external comparisons. The reference point of comparisons changed to the comparative level of alternative relations, which means that the retailers will compare the value of experience in former phases with the expected value of the best alternative manufacturers. The negative results of the comparison will make one side to consider that the other side is the obstacle for it to achieve higher goals, and therefore intends to leave. Conflicts will occur in such process. Even if it does not leave the channel, the relation will drop to recession very quickly. At recession, channel members will show an unharmonious loyalty based on cost shifting. Retailers will choose to focus on the innovation of manufacturers, and actively find new suppliers that can compensate the shifting costs. If it cannot adopt appropriate measures timely to fix the relations and increase the client value, the client relation is possible to end at anytime. The client relation must experience a new process of solving relation conflicts before continuing its development from a trust and behavioral loyalty level.

The features of communication in mature period lead to the rather low standard of communication obstacles, so the influence level of communication obstacles toward channel conflicts is relatively low. Even though, we consider that the communication problem during the mature period is an important issue. Communication contains not only basic information about products trading, but also includes the enterprise culture, strategy, the communication about perception of market environment, pressure from competitions, expectations of roles between manufacturers and retailers. The keys to lower the conflict levels and fix the relation are timely and extensive communication, increased trust, reduced perception and expectation difference, and the timely raise of client value.

\section{Conclusions}

The relation resource among enterprises, which is also referred as the link between enterprises can produce network benefits. One is the opportunity to share resource; the other is to acquire the knowledge-spillover and new technology. Not only new solutions can be known, but also the lesson from failures can be spread through this channel. For trust and learners, on the one hand, the coming up of new and successful solutions depends on the knowledge-spillover benefits from studies among enterprises; on the other hand, the resource-sharing benefits from the trust between enterprises are needed. The two aspects can be described as the idea problem and the action problem (Obstfeld, 2005 [14]). Enterprises can take joint actions easier because of trust, but the production of new designs is limited by thoughts and ideas of both sides. Therefore, enterprises need to improve by studying.

Reducing the perception difference among enterprises is advantageous for sharing confidence, learning knowledge and utilizing resource among enterprises. Knowledge contained in accumulated skills of a professional field is usually invisible and hard for decoding. Information is the basic fact that can be described clearly and passed by simple communication techniques (Kogut and Zander, 1992; Szulanski, 1996) [15]. Resourcesharing benefit is more related to the information sharing based on the trust among enterprises, whereas the knowledge-spillover benefit is more related to the knowledge transmission based on studies among enterprises. The reduction of perception difference can decrease conflicts, and help managers to design efficient strategies for cooperation between enterprises according to the features of products and business partners. In the process of creating relation value by the interaction of enterprise resource, invisible knowledge is hard to be absorbed by an enterprise through a single contact. Reducing the perception difference and establishing effective communication mechanism among enterprises are significant. It is helpful for enterprises to improve innovative abilities when both of them are willing to share information more often, especially to promote the transfer and absorption of invisible knowledge.

\section{Acknowledgements}

This research is supported by BLCU (Beijing Language and Culture University) supported project (supported by the Fundamental Research Funds for the Central Universities) (Approval Number: 15YJ0404). I appreciate the support from BLCU (Beijing Language and Culture University) Business School supported project (supported by the Fundamental Research Funds for the Central Universities) (Approval Number: 15YJ0404). I also appreciate the support from Beijing supporting Central University joint project-Young Talent Plan (Project No. 


\section{YETP0880).}

\section{References}

[1] Rosenberg, L.J. and Stern, L.W. (1970) Toward the Analysis of Conflict in Distribution Channels: A Descriptive Model. European Journal of Marketing, 34, 40-47. http://dx.doi.org/10.2307/1250710

[2] Van De Ven, A.H. and Poole, M.S. (1995) Explaining Development and Changes in Orgnization. Academy of Management Review, 20, 510-540.

[3] Pondy, L.R. (1967) Orgnizational Conflict: Concepts and Models. Administrative Science Quarterly, 12, $296-320$. http://dx.doi.org/10.2307/2391553

[4] Etgar, M. (1979) Sources of Type of Itrachannel Conflict. 55, 61-78.

[5] Stern, L.W., Ansary, A.I. and Coughlan, A.T. (Zhao, P., Translator) (2000) Marketing Channel Management. Tsinghua University Press, Beijing.

[6] Gattorna, J. (1978) Channels of Distribution Conceptualiasation: A State-of-the Art Review. European Journal of Marketing, 12, 471-511. http://dx.doi.org/10.1108/EUM0000000004993

[7] Patrick, L.S. and Emin, B. (1998) An Examination of the Interfirm Power_Conflict Ralationship: The Intervening Role of the Channel Decision Structure. Journal of Retailing, 64, 5-29.

[8] Rosenbloom, B. (2002) Marketing Channels: A Management View. China Machine Press, Beijing.

[9] Zhuang, G.J., Zhou, N. and Zhou, X.L. (2003) Study on the Influence of Perception Difference of Dependence on Channel Conflict. 55, 61-78.

[10] Baron, R.A. and Byrne, D. (1997) Socialpsychology. Allynand Bacon, Mass.

[11] Kelly, H.H. and Jhon, W. (1978) Interpersonal Relation: A Theory of Interdependence. John Wiley \& Sons Inc., New York.

[12] Carr, A.S. and Pearson, J.N. (1999) Strategically Managed Buyer-Seller Relationships and Performance Outcomes. Journal of Operations Management, 14, 497-519. http://dx.doi.org/10.1016/S0272-6963(99)00007-8

[13] Teece, D.J. (2010) Business Models, Business Strategy and Innovation Long Range Planning. 43, $172-194$.

[14] Obstfeld, D. (2005) Social Networks, the Tertius Lungens Orientation, and Involvement in Innovation. Administrative Science Quarterly, 50, 100-130.

[15] Szulanski, G. (1996) Exploring Internal Stickiness: Impediments to the Transfer of Best Practice within the Firm. Strategic Management Journal, 17, 27-43. 